\title{
Simultaneous Estimation and Validation for Determination of Lamivudine and Zidovudine in Human Plasma by LCMS/MS Method
}

\author{
C. PARTHIBAN ${ }^{* \dagger}$, M. BHAGAVAN RAJU ${ }^{\dagger \dagger}$, \\ M. SUDHAKAR ${ }^{\dagger}$, and D. SATHIS KUMAR ${ }^{+\dagger}$ \\ * Department of Pharmaceutical Analysis \\ Malla Reddy College of Pharmacy, Maisammaguda \\ Dhulapally, Secunderabad, Andhra Pradesh, India-500 014 \\ ${ }^{\dagger}$ Department of Pharmaceutical Chemistry \\ C M College of Pharmacy, Maisammaguda \\ Dhulapally, Secunderabad, Andhra Pradesh-500 014, India \\ ${ }^{\dagger}$ Department of Pharmaceutical Analysis \\ Nalanda College of Pharmacy, \\ Nalgonda Andhra Pradesh-508 001, India \\ parthi_0128@yahoo.co.in
}

Received 26 February 2011; Revised 10 May 2011; Accepted 6 June 2011

\begin{abstract}
A high performance liquid chromatographic method with mass detection was developed for determination of Lamivudine and Zidovudine in human plasma. Lamivudine and Zidovudine were extracted from an aliquot of human plasma using Solid Phase Extraction technique, and then injected into a liquid chromatograph equipped with a tandem mass spectrometry detector; quantitation was done by peak area ratio method. A weighted $\left(1 / \mathrm{Conc}^{2}\right)$ linear regression was performed to determine the concentration of analyte. This method demonstrates acceptable performance and is suitable for the determination of Lamivudine and Zidovudine in human plasma over the range of 25.291 to $4046.621 \mathrm{ng} / \mathrm{mL}$ and 23.357 to $4057.141 \mathrm{ng} / \mathrm{mL}$ respectively using Solid Phase Extraction Procedure. As all the values obtained were within the limits as specified, the method has been successfully used to analyse the human plasma containing Lamivudine and Zidovudine with good recoveries and proved to be robust.
\end{abstract}

Keywords: Lamivudine, Zidovudine, Abacavir, LC/MS/MS, Validation.

\section{Introduction}

Lamivudine, ([2R-cis]-4-amino-1-[2-(hydroxymethyl)-1,3-oxathiolan-5-yl]-2(1H)-pyrimidinone $)^{1}$ and Zidovudine, (3'-azido-3'-deoxythymidine) $)^{2}$ are well established anti HIV agents ${ }^{3}$. Lamivudine is official in $\mathrm{IP}^{3}$ and USP. Zidovudine is official in IP, $\mathrm{BP}^{4}$ and $\mathrm{EP}^{5}$. Most antiviral agents are used as part of combination therapy for the treatment of AIDS. Since there are several generic combination products available in the worldwide market for 
polytherapy, an assay method for the estimation of these drugs simultaneously is of utility. $\mathrm{E} \mathrm{K}$ Kano et al, evaluated the lamivudine in human plasma by HPLC, finally they concluded that the two lamivudine formulations are bioequivalent in their rate and extent of absorption and thus, may be used interchangeably ${ }^{6}$. Bengi Uslu et al, determined the binary mixture of lamivudine and zidovudine by first derivative spectrophotometric, first derivative of the ratio-spectra and high-performance liquid chromatography-UV methods ${ }^{7}$. Hedaya MA et al, developed a sensitive and specific HPLC method for the determination of zidovudine and zidovudine glucuronide in plasma and urine ${ }^{8}$. The literature review reveals that there are few HPLC ${ }^{9,10}$, UV methods have been reported for this combination, but no LCMS/MS method has so far been reported for the simultaneous determination of these drugs in pharmaceutical preparations as well as biological solution. The objective of this work is to validate specific LC/MS/MS method for the determination of Lamivudine and Zidovudine in human plasma for bioequivalence and bioavailability study of Lamivudine and Zidovudine.

\section{Experimental}

Lamivudine (LAM), Zidovudine (ZID) and Abacavir (ABA) were obtained from local market. The HPLC grade Acetonitrile, Methanol, and Formic acid from Rankem (New Delhi, India) and all other chemicals used were of pharmaceutical or analytical grade from Rankem. HPLC grade water was prepared using Millipore purification system. An API 2000 LC/MS/MS system equipped with a pump (Shimadzu LC 2OADvp), an auto Sampler (Shimadzu SIL-HTc), analytical column Hypurity advance (Thermo electron) $\mathrm{C}_{18}$ (50 mmx4.6 mm, 5p.m) and data acquisition system and quantitation program (Applied Biosystems "Analyst" version 1.4.1) was used for the quantitative determination of LAM and ZID in human plasma. The interface used with the API $2000 \mathrm{LC} / \mathrm{MS} / \mathrm{MS}$ was a Turbo ion spray ${ }^{\circledR}$. The Positive ions were measured in MRM mode for the two analytes (LAM and ZID) and the internal standard (ABA).

\section{Preparations}

A stock solution of $1000 \mu \mathrm{g} / \mathrm{mL}$ each of LAM, ZID, and Internal standard ABA were prepared using methanol (Diluent-1). The stock solution was protected from light using aluminum foil and stored in the refrigerator at $2-10^{\circ} \mathrm{C}$. From the above stock solution $100 \mu \mathrm{g} / \mathrm{mL}$ each of LAM, ZID were prepared using $60 \%$ methanol-water solution (Diluent-2). For Internal standard ABA, $6 \mu \mathrm{g} / \mathrm{mL}$ was prepared using diluent-2 from the above internal stock solutions.

\section{Calibration curve standards and quality control samples}

The LAM and ZID working solution standards for the plasma calibration curve were prepared by diluting stock of LAM and ZID with diluent-2 to yield the required concentrations of $25.291,50.583,101.166,303.497,809.324,1618.648,2427.973$, 3034.966 , and $4046.621 \mathrm{ng} / \mathrm{mL}$ for LAM and for ZID was composed of 23.357, 50.714, $101.429,304.286,811.428,1622.856,2434.285,3042.856,4057.141 \mathrm{ng} / \mathrm{mL}$ and two blanks with internal standard and two blanks without internal standard were analysed with each run of validation. The standards were prepared fresh with each batch run of the stability exercises of validation.

Quality Control (QC) samples were prepared in bulk by mixing 0.2 parts of the respective working QC solutions each of LAM and ZID with 9.6 parts of blank human plasma and were stored at $-20^{\circ} \mathrm{C} \pm 15^{\circ} \mathrm{C}$. Six sets of QC samples at concentrations of low $75.844 \mathrm{ng} / \mathrm{mL}$, medium $1921.391 \mathrm{ng} / \mathrm{mL}$ and high $3236.026 \mathrm{ng} / \mathrm{mL}$ concentrations for LAM 
and low $76.057 \mathrm{ng} / \mathrm{mL}$, medium $1926.765 \mathrm{ng} / \mathrm{mL}$ and high $3245.079 \mathrm{ng} / \mathrm{mL}$ for ZID were assayed with each batch run of the validation. Three sets of QC samples (LQC \& HQC) were also stored at $-70^{\circ} \mathrm{C} \pm 15^{\circ}$ to evaluate long term stability. $200 \mu \mathrm{L}$ of plasma transferred into RIA vials, spiked with $20 \mu \mathrm{L}$ of internal standard $(6 \mu \mathrm{g} / \mathrm{mL})$ and mixed on vortexer.

The plasma mixture was loaded on previously conditioned (conditioned with $2 \mathrm{~mL}$ of methanol followed by $2 \mathrm{~mL}$ of Milli-Q water) Celerity deluxe cartridges (Orochem, India). The cartridges were washed with $1.0 \mathrm{~mL}$ of Milli-Q water followed by $5 \%$ methanol in Milli$\mathrm{Q}$ water, elute with $1.0 \mathrm{~mL}$ of methanol. The sample was evaporated to complete dryness under a steam of nitrogen gas at $\leq 40^{\circ} \mathrm{C}$. The residue was reconstituted with $2 \mathrm{~mL}$ of reconstitution solution. Load $200 \mu \mathrm{L}$ of reconstituted samples into the HPLC vial and inject $5 \mu \mathrm{L}$ of sample into the LC/MS/MS system.

\section{Validation}

The described method has been validated for the assay of LAM \& ZID using following parameters.

\section{Precision and accuracy}

Intra day accuracy and precision evaluation were assessed by analyzing two calibration curves and three sets of QC samples, six replicates each of the LQC, MQC and HQC run on the same day. Inter Day accuracy and precision evaluation were assessed by analyzing five sets of calibration curves and three sets of QC samples, six replicates each of the LQC, MQC and HQC on different days. The precision and accuracy at the ULOQ $(4045.033 \mathrm{ng} / \mathrm{mL})$ and the LLOQ $(25.281 \mathrm{ng} / \mathrm{mL})$ for LAM and at ULOQ $(4056.348 \mathrm{ng} / \mathrm{mL})$ and the LLOQ $(25.352 \mathrm{ng} / \mathrm{mL})$ for ZID were evaluated. The percentage recovery of LAM and ZID was determined by comparing the mean peak area of LAM and ZID in extracted LQC, MQC and HQC samples with freshly prepared unextracted LQC, MQC and HQC samples respectively.

\section{Blank matrix specificity and limit of quantitation}

During the selectivity run, an LLOQ standard was extracted and injected. The responses for the blank plasma from the eight donors were compared to the LLOQ standard of the two analytes and internal standard.

\section{Ruggedness}

Ruggedness experiment with different analyst, the sample was processed. The run consisted of a calibration curve standard and six set at each of low (LQC), medium (MQC) and high (HQC) concentrations. Ruggedness experiment on different column, sample was processed on different columns with same make and specification. Concentrations were calculated to determine precision and accuracy.

\section{Dilution integrity}

Dilution Integrity experiment was carried out at six replicate of five times diluted ( 1 in 5 dilutions) and ten times diluted of approx. 2.5x ULOQ (1 in 10dilution) samples were prepared and concentrations were calculated including the dilution factor against the freshly prepared calibration curve. Samples were prepared at LQC and HQC level in six different lots of human plasma sources. A calibration curve and six replicates of LQC and HQC samples for each matrix were freshly processed and analyzed in single run. Percentage Bias was calculated for each matrix. 


\section{Partial volume analysis}

In Partial Volume Analysis, Low (LQC) \& high (HQC) QC samples were taken out of freezer and allowed them to thaw and these samples were diluted with pre-checked interference-free plasma to make six sets each $1 / 2,1 / 4^{\text {th }}$ concentration. Back-calculated concentrations were determined against the standard curve using the dilution factor and the results were compared with those from normal volumes.

\section{Partial and whole batch re-injection reproducibility}

To evaluate the partial re-injection reproducibility experiment samples were kept in the auto sampler at $5^{\circ} \mathrm{C}$, for approx $2.0 \mathrm{hrs}$ and re-injected. Concentrations were calculated to determine precision and accuracy after re-injection. To evaluate the whole batch re-injection reproducibility experiment samples were kept on bench at room temperature for approx 20 hrs after the initial analysis and were re-injection again after approx $20 \mathrm{~h}$. Concentrations were calculated to determine precision and accuracy after re-injection.

\section{Stability studies}

\section{Bench top stability}

LAM and ZID (at room temperature for 27 hours $15 \mathrm{~min}$ ) LQC and HQC samples were spiked in human plasma and were kept at room temperature for 27 hours 15 min and were processed and analyzed along with freshly prepared calibration standards, comparison LQC and HQC samples. Concentrations were calculated to determine mean \% change during stability period.

\section{Stability of analyte}

To qualify the stability of analytes in the biological matrix during transportation of the samples, the dry ice stability (coolant) was determined. Six sets of samples each of low (LQC) and (LQC) were kept in dry ice for approximately 27 hours. Stability samples at each level low (LQC) and high (HQC) were processed and they were analyzed along with freshly processed calibration and comparison samples (freshly processed LQC and HQC).

\section{Dry extract stability}

The stability of LAM and ZID (after extraction and sample preparation in the dry extract condition) during storage in the refrigerator $\left(2-10^{\circ} \mathrm{C}\right)$ was documented by comparison of the concentrations of extracted replicates to the comparison samples (freshly processed LQC and HQC) concentrations.

The six sets of extracted plasma QC samples at concentrations of low (LQC) and high (HQC) for LAM and ZID were kept in a refrigerator at $2-10^{\circ} \mathrm{C}$ for approximately 26 hours after extraction in dry extract conditions and prior to analysis. They were then analysed using a fresh standard curve and freshly processed comparison samples.

\section{Freeze and thaw stability}

Samples were prepared at LQC and HQC levels, aliquot and frozen at $-20 \pm 5^{\circ} \mathrm{C}$. Six samples from each concentration were subjected to three freezes and thaw cycles (stability samples).

These samples were processed and analysed along with freshly prepared calibration standards, LQC and HQC samples (comparison samples). Concentrations were calculated to determine mean $\%$ change after four cycles. 


\section{In- Injector stability}

LQC and HQC samples were prepared and processed. These processed samples were analyzed and kept in auto sampler for 31 hours at $5^{\circ} \mathrm{C}$ and analyzed along with freshly prepared calibration standard samples. Concentrations were calculated to determine mean $\%$ change during stability period.

\section{Stock solution stability}

Stock solution stability was determined by comparing the peak areas of freshly prepared solutions (comparison samples) with stability samples. Main stock solutions of LAM, ZID \& ABA were freshly prepared and aliquots of stocks were kept at room temperature for 8 hours (stability sample). Aqueous equivalent highest calibration standard of LAM, ZID \& ABA were prepared from the stability samples and analyzed. Areas of stability samples and freshly prepared samples were compared to determine mean \% change during stability period.

For the Long term stock solution stability, the stock solution of LAM and ZID were prepared on 03/12/2009 (MD), 07/12/2009 (MV) and stored in the refrigerator at $2-10^{\circ} \mathrm{C}$ for 10 and 6 days respectively. Internal Standard (ABA) was prepared on 03/12/2009 (MD), $07 / 12 / 2009$ (MV) and stored in the refrigerator at $2-10^{\circ} \mathrm{C}$ for 10 and 6 days respectively. Stock solution of LAM, ZID \& ABA was compared against fresh stock solution prepared on 13/12/2009.

For the Long term stock solution stability in diluent-2, the working solutions of LAM, ZID were prepared on 07/12/2009 and stored in the refrigerator at $2-10^{\circ} \mathrm{C}$ for 6 days. Working solutions of LAM \& ZID were compared against fresh stock solution prepared on $13 / 12 / 2009$.

\section{Injector carry over effect for analyte and internal standard}

Carryover test was performed in the following sequence. Mobile Phase (MP) $\rightarrow$ Aqueous mixture $\rightarrow \mathrm{MP} \rightarrow \mathrm{MP} \rightarrow$ Plasma Blank (PLB) $\rightarrow \mathrm{CS} 9$ (Highest Calibration) $\rightarrow \mathrm{MP} \rightarrow \mathrm{MP}$ $\rightarrow \mathrm{PLB} \rightarrow \mathrm{CS} 9 \rightarrow \mathrm{PLB} \rightarrow \mathrm{MP}$.

\section{Results and Discussion}

The retention times of LAM, ZID and ABA are approximately 1.13, 1.51, and 0.97 minutes respectively. The overall chromatographic run time is 3.5 minutes. Mass spectrum of LAM, ZID and ABA were present in Figure 1. During the validation, blank plasma samples from eight donors were evaluated and all plasma lots were found to be satisfactory. Figure 2 expressed that there were no interfering peaks in the blank plasma at the retention times of LAM and ZID or internal standard as seen in the chromatograms.

\section{Calibration curve method}

Table 1 expressed the summary of calibration curve method. Calibration curves were found to be consistently accurate and precise for LAM and ZID over 25.291 to $4046.621 \mathrm{ng} / \mathrm{mL}$ and 23.357 to $4057.14 \mathrm{ng} / \mathrm{mL}$ respectively for calibration range. The correlation coefficient was greater than or equal to 0.9985 for LAM and 0.9991 for ZID. Table 2 expressed the back calculations which were made from the calibration curves to determine LAM and ZID concentrations of each calibration standard. 


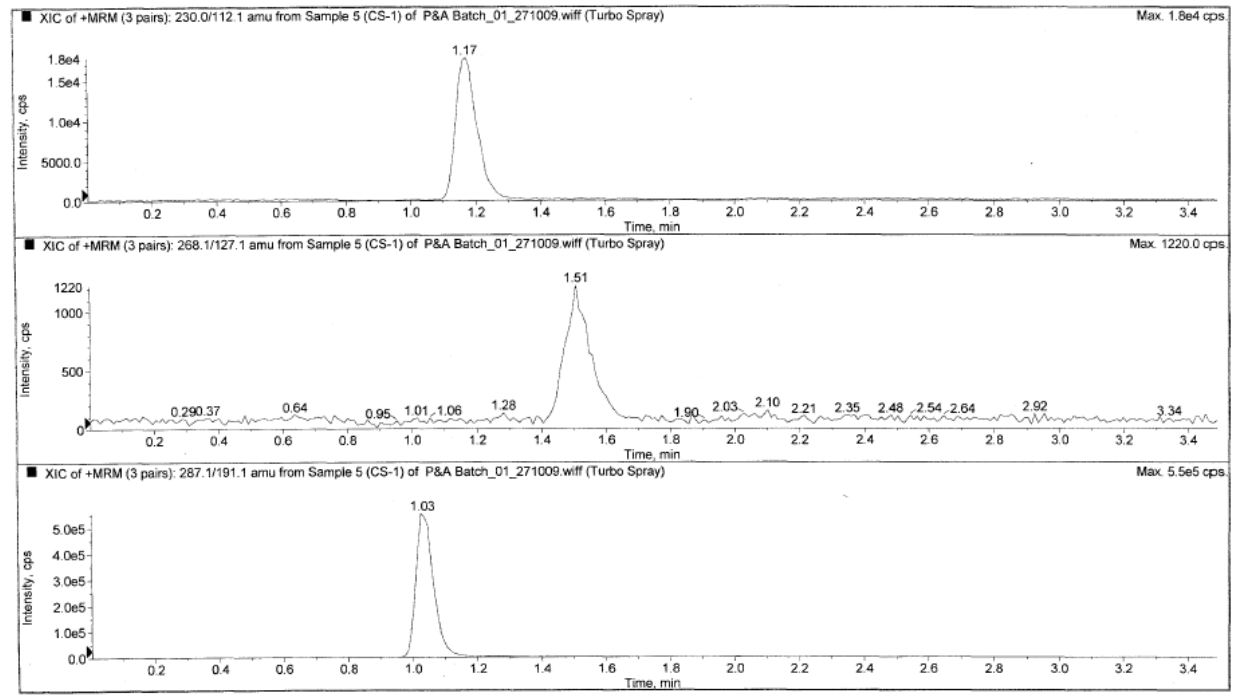

Figure 1. Spectrum for Lamivudine, Zidovudine, and Abacavir.

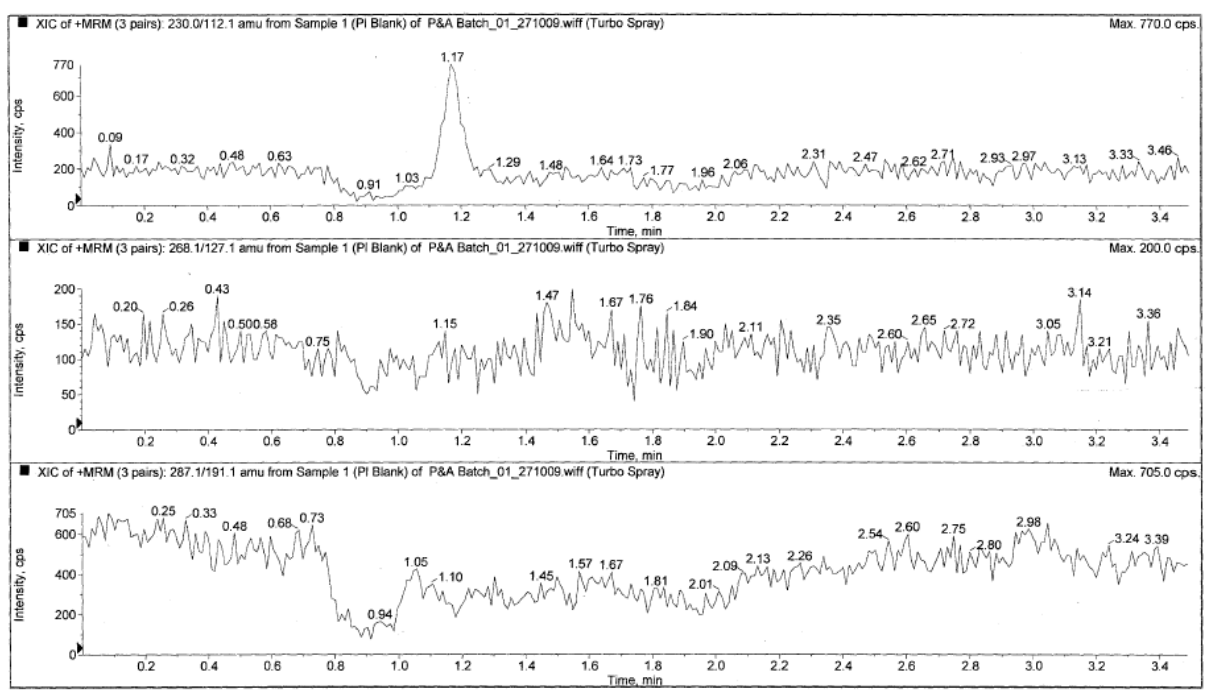

Figure 2. Spectrum for plasma interference with samples and standard.

Table 1. Summary of calibration curve method.

\begin{tabular}{|c|c|c|}
\hline \multirow{2}{*}{$\begin{array}{l}\text { Calibration curve } \\
\text { method }\end{array}$} & \multicolumn{2}{|c|}{ In human plasma } \\
\hline & Lamivudine & Zidovudine \\
\hline Y-Intercept & $0.001953 \pm 0.0005$ & $0.00011 \pm 0.000009$ \\
\hline $\begin{array}{l}\text { Slope } \\
\text { Coefficient of } \\
\text { regression }\end{array}$ & $\begin{array}{c}0.00049 \pm 0.000002 \\
0.99855 \pm 0.0005\end{array}$ & $\begin{array}{c}0.000008 \pm 0.0000008 \\
0.9991 \pm 0.0004\end{array}$ \\
\hline $\mathrm{n}=6$, Mean \pm S.D & & \\
\hline
\end{tabular}


Table 2. Back-Calculated Standard Concentrations for LAM and ZID in human plasma.

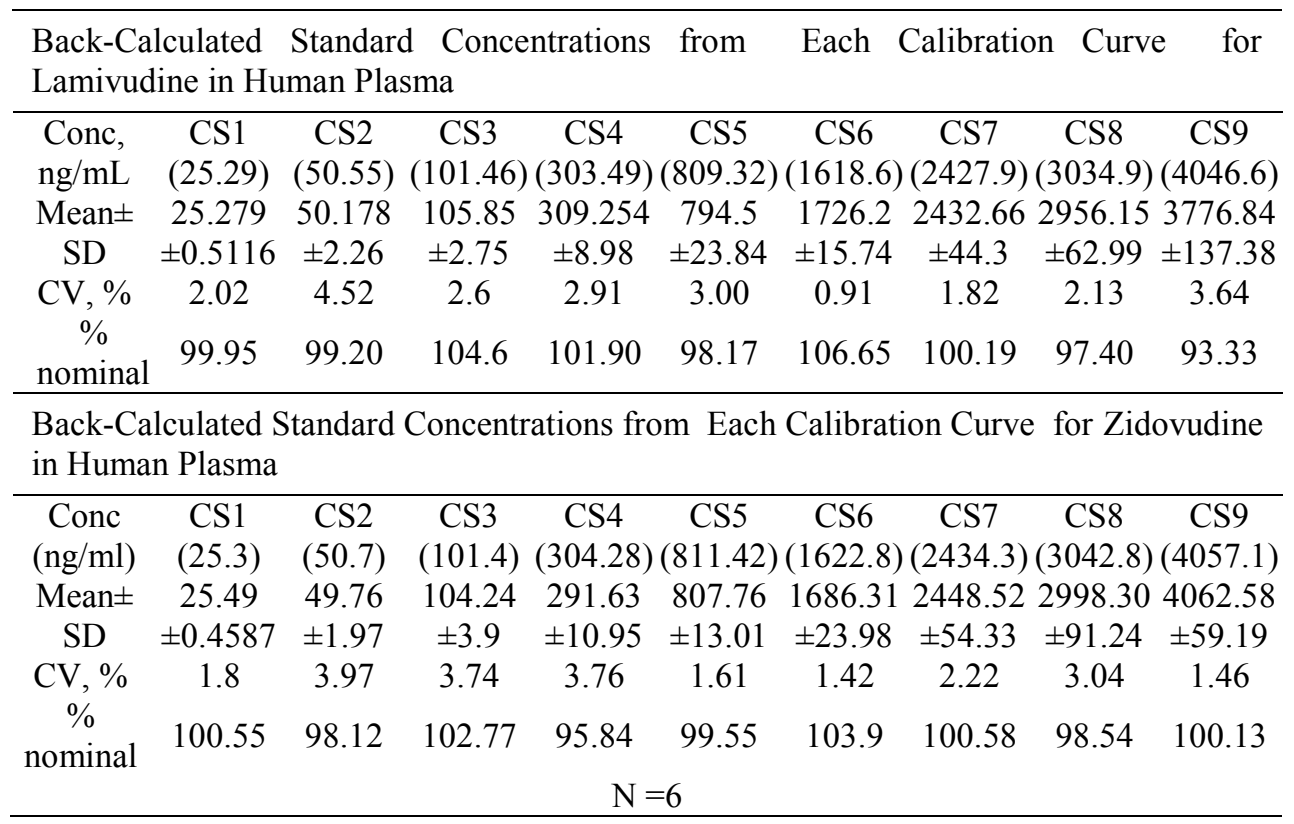

\section{Intra and inter day precision and accuracy}

The Intra day percentages of nominal concentrations for LAM and ZID were ranged between 91.96 to $104.29 \%$ and 94.18 to $102.08 \%$ respectively. The Intra day percentage coefficient of variation is 4.01 to $4.44 \%$ for LAM and 2.58 to $3.60 \%$ for ZID. The Inter Day percentages of nominal concentrations for LAM and ZID were ranged between 91.84 to $103.55 \%$ and 94.21 to $100.83 \%$ respectively. The Inter Day percentage coefficient of variation is 3.73 to $5.15 \%$ for LAM and 3.21 to $3.79 \%$ for ZID. Inter day and intra day data were present in Table 3. The within-run precision where present in Table $4 .(\% \mathrm{CV})$ at the ULOQ and LLOQ was $3.92 \%$ and $2.73 \%$ respectively for LAM and $2.88 \%$ and $3.82 \%$ respectively for ZID. The Intra day accuracy at the ULOQ and LLOQ was $92.61 \%$ and $89.54 \%$ respectively for LAM and $103.76 \%$ and $92.65 \%$ respectively for ZID.

\section{Recovery}

The mean \% recovery for LQC, MQC and HQC samples of LAM were 65.60, 71.02, and 74.83 respectively. The mean recovery of LAM across QC levels was $70.48 \%$. The mean $\%$ recovery for LQC, MQC and HQC samples of ZID were 77.43, 81.70, and 82.58 respectively. The mean recovery of ZID across QC levels is $80.57 \%$. For the internal standard, mean peak area of 18 extracted samples was compared to the mean peak area of 18 unextracted ABA solutions. The mean percentage recovery was $97.30 \%$.

\section{Blank matrix specificity and Limit of quantitation}

No significant response ( $\leq 20 \%$ for the analyte response and $\leq 5 \%$ of the internal standard response) was observed at the retention time of the analytes or the internal standard in blank plasma as compared to the LLOQ standard. LLOQ response mean value was 41009, 7505 for LAM and ZID respectively and LLOQ, S/N Ratio mean value were 282.68 and 63.63 for LAM and ZID respectively. 
Table 3. Inter day and intra day precision and accuracy for LAM and ZID.

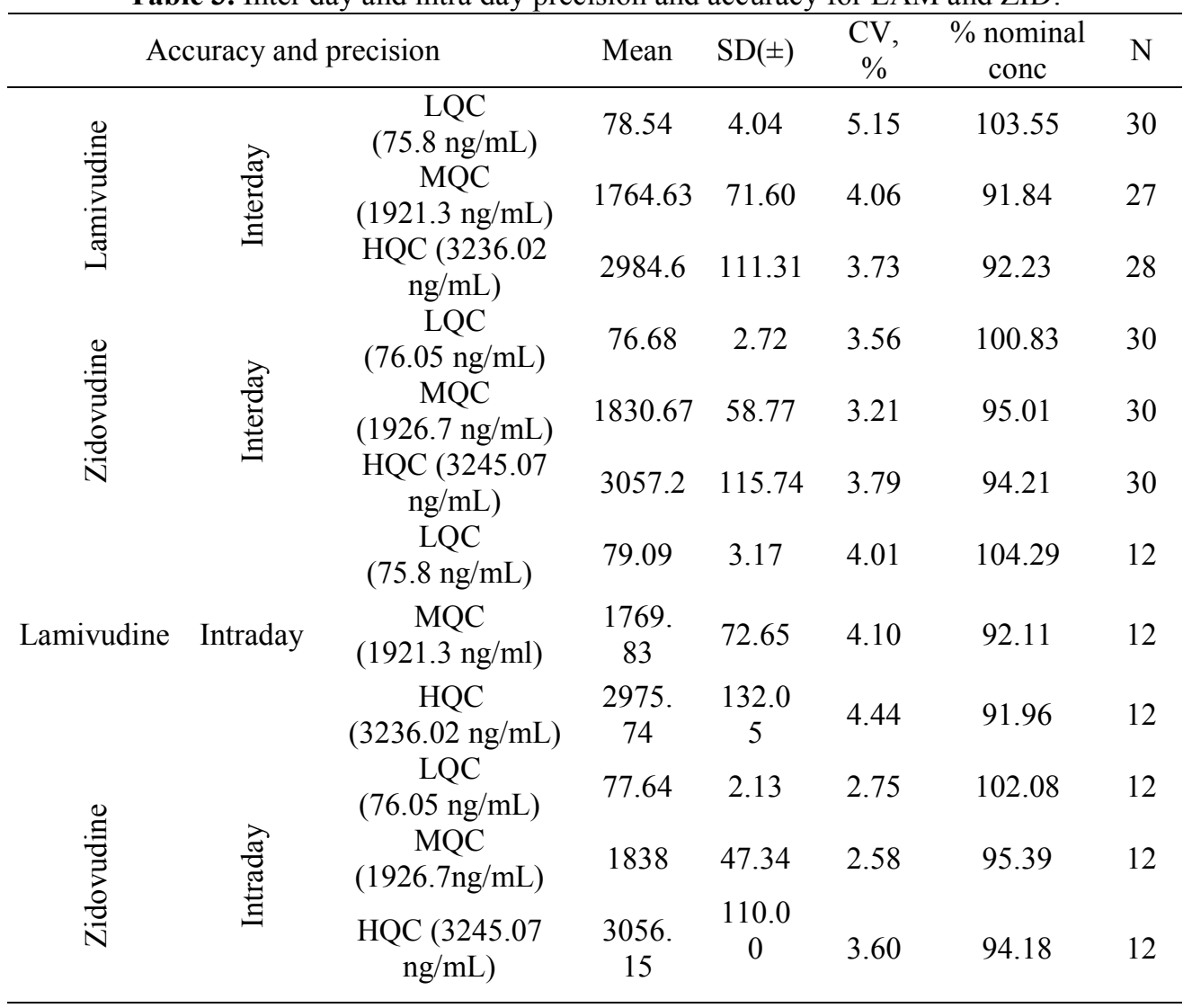

Table 4. Within run accuracy analysis for LAM and ZID in human plasma.

\begin{tabular}{|c|c|c|c|c|c|c|c|c|c|c|}
\hline & \multicolumn{5}{|c|}{ Lamivudine } & \multicolumn{5}{|c|}{ Zidovudine } \\
\hline $\begin{array}{l}\text { Conc } \\
\mathrm{ng} / \mathrm{mL}\end{array}$ & $\begin{array}{c}\text { LLOQ } \\
(25.2)\end{array}$ & $\begin{array}{c}\text { QC LOY } \\
(64.4)\end{array}$ & $\begin{array}{l}\text { QC MED } \\
(1921.3)\end{array}$ & $\begin{array}{c}\text { QC } \\
\text { HIGH } \\
(3236.0)\end{array}$ & $\begin{array}{c}\text { ULOQ } \\
(4045.0)\end{array}$ & $\begin{array}{c}\text { LLOQ } \\
(25.3)\end{array}$ & $\begin{array}{c}\text { QC LOW } \\
(76.0)\end{array}$ & $\begin{array}{c}\text { QC } \\
\text { MED } \\
(1926.7\end{array}$ & $\begin{array}{c}\text { QC } \\
\text { HIGH } \\
(3245.0)\end{array}$ & $\begin{array}{c}\text { ULOQ } \\
(4056.3)\end{array}$ \\
\hline Mean & 22.637 & 75.15 & 1699.18 & 2896.82 & 3746.08 & 23.48 & 76.17 & 1822.85 & 3074.35 & 4208.79 \\
\hline$\pm \mathrm{SD}$ & 0.6178 & 3.25 & 58.53 & 101.74 & 146.72 & 0.89 & 1.44 & 63.51 & 162.69 & 121.19 \\
\hline $\mathrm{CV}, \%$ & 2.73 & 4.33 & 3.44 & 3.51 & 3.92 & 3.82 & 1.90 & 3.48 & 5.29 & 2.88 \\
\hline $\begin{array}{c}\% \\
\text { nominal }\end{array}$ & 89.54 & 99.10 & 88.44 & 89.52 & 92.61 & 92.65 & 100.15 & 94.61 & 94.74 & 103.76 \\
\hline
\end{tabular}

\section{Ruggedness}

The precision $(\% \mathrm{CV})$ of LAM QC samples was within the range of 4.32 to $4.56 \%$ and the accuracy was within the range of 90.47 to $102.42 \%$ and the precision and accuracy for ZID. QC samples were within the range of 2.81 to $3.96 \%$ and 93.29 to $100.95 \%$ respectively. These results indicated that the method was rugged and reproducible by different analyst. LAM and ZID results were found to be reproducible on different column with same make and specification. The precision (\%CV) of LAM QC samples was within the range of 2.24 to $5.37 \%$ and the accuracy was within the range of 89.94 to $105.04 \%$. The precision (\%CV) 
of ZID QC samples was within the range of 2.97 to $4.50 \%$ and accuracy was within the range of 91.29 to $101.77 \%$.

\section{Dilution integrity}

The percentage accuracy of LAM nominal concentrations ranged between 93.73 to 100.11 and 92.90 to $109.04 \%$ for 1 in 5 dilutions and 1 in 10 dilutions. The percentage coefficient of variation was 2.37 to $6.03 \%$. The percentage accuracy of ZID nominal concentrations ranged between 90.82 to 99.26 and 87.22 to 103.65 for 1 in 5 and 1 in 10 Dilutions. The percentage coefficient of variation was 3.29 to $6.13 \%$.

\section{Matrix effect}

No significant matrix effect was found in different sources of human plasma tested.

\section{Partial volume analysis}

The precision (\%CV) for $1 / 2$ and $1 / 4$ th concentration of LAM low (LQC) and high (HQC) was $3.23 \& 3.35 \%$ and $3.53 \& 1.19 \%$ respectively and accuracy was $93.42 \& 98.84$ and $93.29 \& 99.01 \%$ respectively. The precision $(\% \mathrm{CV})$ for $1 / 2$ and $1 / 4^{\text {th }}$ concentration of ZID low (LQC) and high (HQC) was $2.28 \& 2.19 \%$ and $5.57 \& 1.31 \%$ respectively and accuracy was $93.51 \& 92.78$ and $96.93 \& 87.87 \%$ respectively.

\section{Partial re-injection reproducibility}

There is no significant variability in the concentration of analyte after re-injection (approx $2.0 \mathrm{hrs})$ in auto sampler at $5^{\circ} \mathrm{C}$. The precision $(\% \mathrm{CV})$ and accuracy of LAM QC samples in re-injection was between 2.07 to $4.06 \%$ and 90.46 to $100.91 \%$ while for ZID QC samples in re-injection was between 2.78 to $4.53 \%$ and 90.27 to $94.10 \%$.

\section{Whole batch re-injection reproducibility}

LAM and ZID were found to be stable at room temperature post extraction (in reconstitution solution) for approx $20 \mathrm{hrs}$ and reproducible after re-injection. The precision $(\% \mathrm{CV})$ and accuracy of LAM QC samples in re-injection was between 2.99 to $4.70 \%$ and 92.60 to $108.92 \%$ respectively, while for ZID QC samples in re-injection were between 2.69 to $7.30 \%$ and 93.39 to $102.80 \%$ respectively.

\section{Stability studies}

\section{Bench top stability}

LAM and ZID was found to be stable in LQC and HQC samples for 27 hours 15 min at room temperature with mean \% change of -1.12 and -0.54 respectively for LAM and mean $\%$ change of 1.62 and -1.31 respectively for ZID.

\section{Stability of analyte in biological matrix in dry ice}

The percent change for LAM was 0.82 and $4.05 \%$ at low (LQC) and high (HQC) from the comparison samples and for ZID at low (LQC) and high (HQC) from the comparison samples was 2.80 and $2.90 \%$ respectively. Results of Dry ice stability studies show that the plasma samples of LAM and ZID were found to be stable for approximately 27 hours in dry ice to cover the exposed duration of samples during shipment of the clinical samples.

\section{Dry extract stability}

The percentage change for LAM was 1.07 and $1.24 \%$ at low (LQC) and high (HQC) from the comparison samples and for ZID at low (LQC) and high (HQC) from the comparison samples was 1.94 and $2.81 \%$ respectively. Refrigerator $\left(2-10^{\circ} \mathrm{C}\right)$ stability studies results 
show that LAM and ZID in dry extract form were stable during storage in the refrigerator $\left(2-10^{\circ} \mathrm{C}\right)$ for at least 26 hours.

Freeze and thaw stability of LAM and ZID (after 4 cycle at $-20 \pm 5{ }^{\circ} \mathrm{C}$ )

LAM and ZID was found to be stable in LQC and HQC samples after 4 cycles at $-20 \pm 5^{\circ} \mathrm{C}$ with mean $\%$ change of -2.34 and -1.52 respectively for LAM and with mean $\%$ change of 4.76 and -0.56 respectively for ZID.

In-injector stability of LAM and ZID at $5{ }^{\circ} \mathrm{C}$ in auto sampler

LAM \& ZID were found to be stable in LQC and HQC samples for 31 hours at $5^{\circ} \mathrm{C}$ in auto sampler with mean \% change of 6.69 and 9.27 respectively for LAM and with mean \% change of -0.75 and -0.64 respectively for ZID.

Stock solution stability of LAM, ZID and ABA

LAM \& ZID stock solution were found to be stable at room temperature for 8 hours with mean \% change at LQC was $1.92 \% \&$ at HQC was $1.33 \%$ and mean $\%$ change at LQC was $4.48 \%$ \& HQC $0.76 \%$ respectively. ABA stock solution was found to be stable at room temperature for 8 hours with mean $\%$ change of 0.56 . The Percentage change for LAM was $0.80 \%$ and $-1.62 \%$ and for ZID was $3.76 \%$ and 0.80 for 10 and 6 days respectively. The Percentage change for ABA was $-1.06 \%$ and -1.48 for 10 and 6 days respectively. These results indicate that the stock solutions for LAM, ZID \& ABA were stable for at least 10 days in the refrigerator at $2-10^{\circ} \mathrm{C}$. The Percentage change for the LAM \& ZID was $-1.20 \%$, and $-0.96 \%$ for 6 days. The percentage change for the ABA was -2.05 for 6 days. No significant injector carry over was observed for LAM, ZID and ABA. The results indicated that there was no effect of injector carry over.

\section{Conclusion}

The method stands validated and is suitable for simultaneous estimation of plasma Lamivudine and Zidovudine concentrations as a single analytical run, in clinical samples from Bioequivalence studies following oral administration of Lamivudine and Zidovudine fixed dose $(150 / 300 \mathrm{mg})$ tablets in healthy human subjects.

\section{References}

1. The United state Pharmacopoeia, Convention Inc., Twin brook Parkway, Rockville, 2003, 26.

2. The Merck Index, $13^{\text {th }}$ Edn., Merck \& Co., Inc., Whitehouse Station. NJ, 2001.

3. Indian Pharmacopoeia - Addendum, The Controller of Publications, New Delhi, 2002.

4. British Pharmacopoeia, Vol. II, Her Majesty's Stationary Office, London, 2003.

5. European Pharmacopoeia, $4^{\text {th }}$ Edn., Council of Europe, Strasbourg, 2002.

6. Eunice Kazue Kano, Cristina Helena dos Reis Serra, Eunice Emiko Mori Koono, Simone Schramm Andrade and Valentina, Int J Pharm., 2005, 297(1-2), 73-79.

7. Bengi Uslu and Sibel A. Ozkan, Anal Chim Acta, 2002, 466 (1), 175-185.

8. Hedaya M A and Sawchuk R J, International Conference AIDS University of Minnesota, Minneapolis, Minnesota, USA. 1989 Jun 4-9; 5: 278 (abstract no. M.B.P.339).

9. Jayakar B, Kumar M, Saravanan C and Kumudhavalli M V, J Chem Pharm Res., 2010, 1, 478-481.

10. Nandini Pai and A D Desai, Indian J Pharmceutical Sci., 2007, 69s(1), 118-120. 


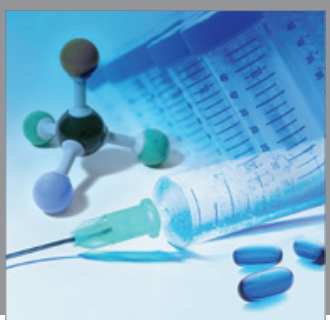

International Journal of

Medicinal Chemistry

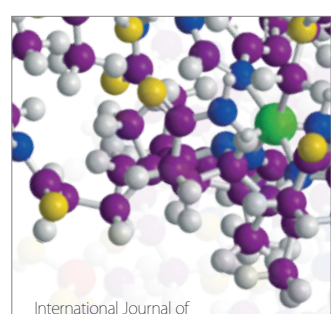

Carbohydrate Chemistry

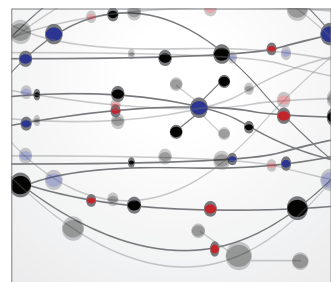

The Scientific World Journal
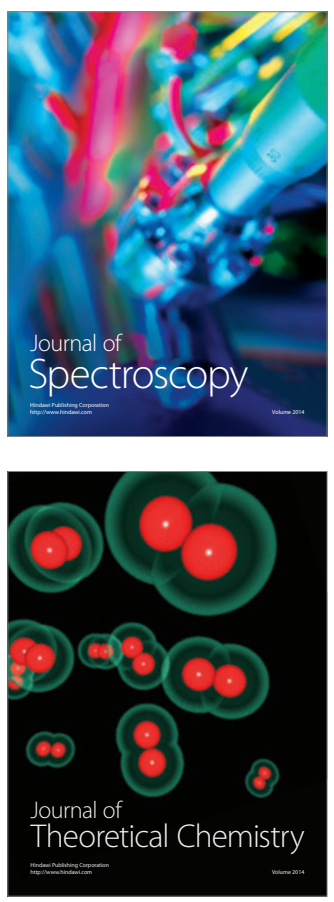
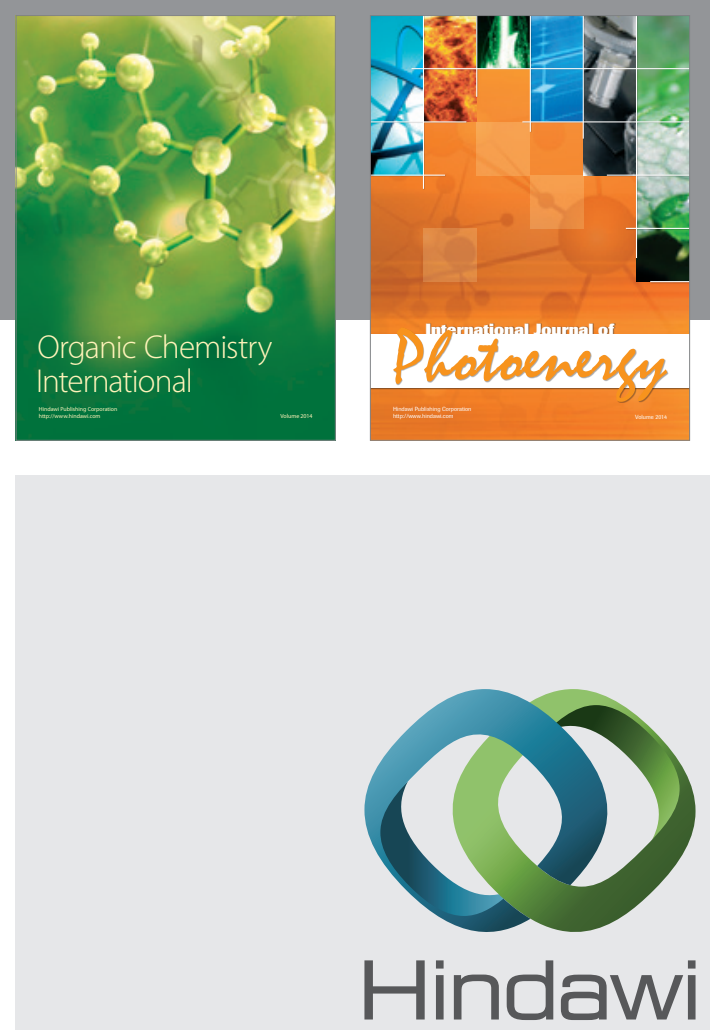

Submit your manuscripts at

http://www.hindawi.com
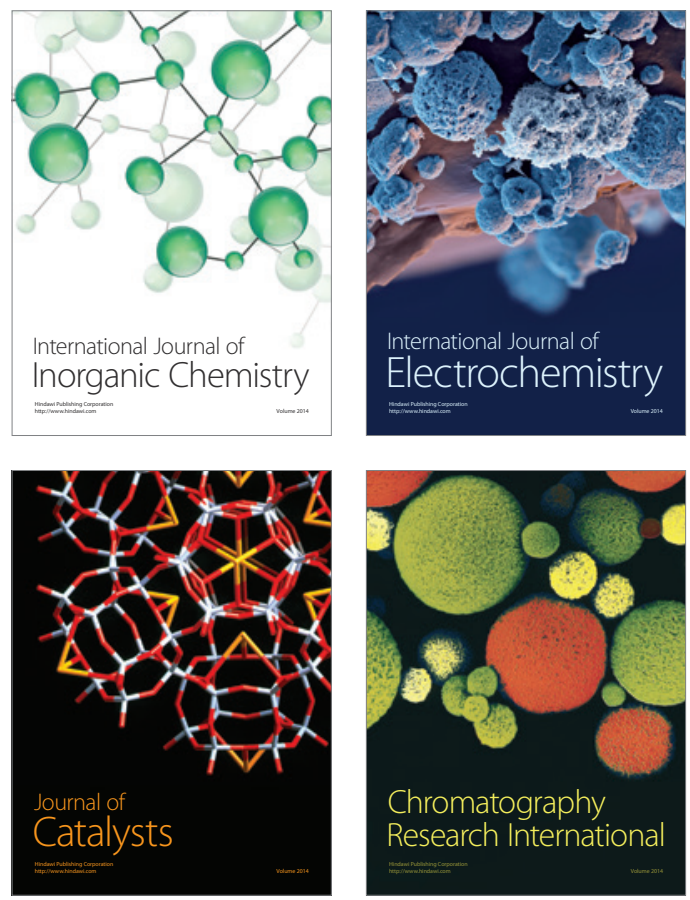
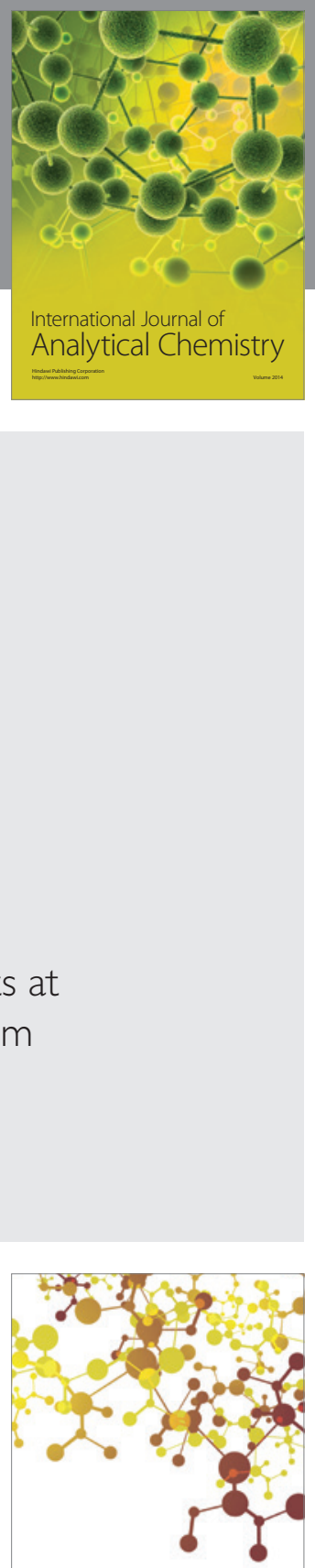

Journal of

Applied Chemistry
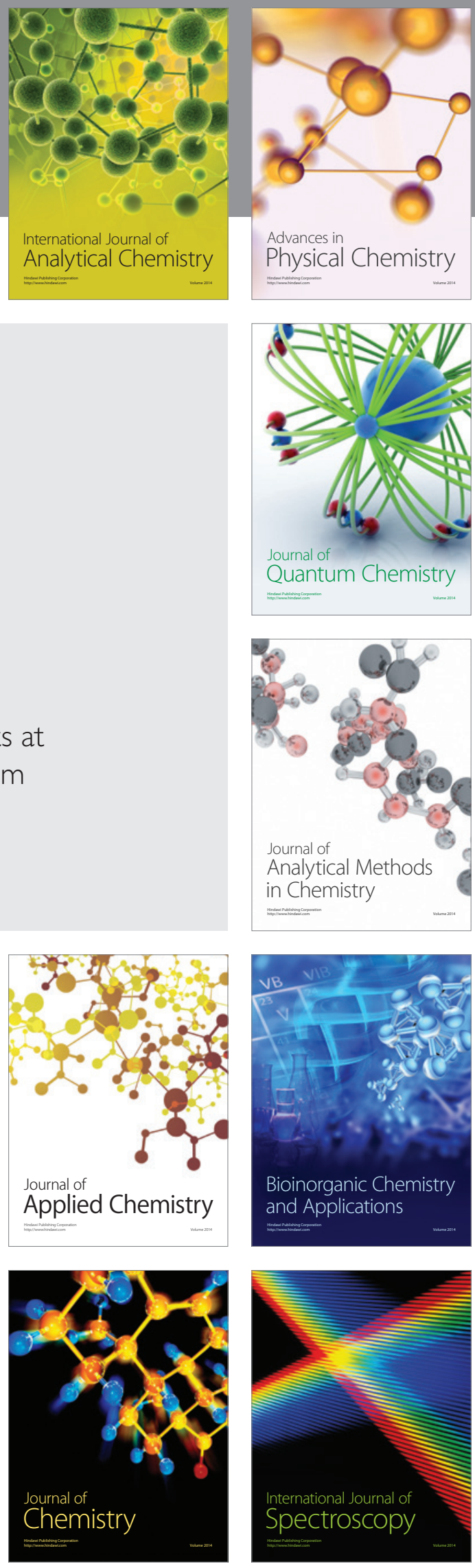